\title{
Vincent Morley, The Popular Mind in Eighteenth- century Ireland, Cork University Press, 2017
}

\section{Thierry Robin}

\section{OpenEdition}

\section{Journals}

\section{Édition électronique}

URL : http://journals.openedition.org/etudesirlandaises/5416

DOI : 10.4000/etudesirlandaises.5416

ISSN : 2259-8863

\section{Éditeur}

Presses universitaires de Caen

\section{Édition imprimée}

Date de publication : 29 novembre 2017

Pagination : 168-170

ISBN : 978-2-7535-7388-8

ISSN : 0183-973X

\section{Référence électronique}

Thierry Robin, « Vincent Morley, The Popular Mind in Eighteenth-century Ireland, Cork University Press, 2017 », Études irlandaises [En ligne], 42-2 | 2017, mis en ligne le 29 novembre 2017, consulté le 24 septembre 2020. URL : http://journals.openedition.org/etudesirlandaises/5416 ; DOI : https://doi.org/ 10.4000/etudesirlandaises.5416

\section{(c) (†) 8 ()}

Études irlandaises est mise à disposition selon les termes de la Licence Creative Commons Attribution - Pas d'Utilisation Commerciale - Partage dans les Mêmes Conditions 4.0 International. 
the topics covered is the antiquarian or historically-minded search for Irish footprints on the Continent (Fontenoy, Montorio, etc.). In an era when women's sphere was thought to be domestic, their travel opportunities were accordingly more limited. Yet, Irish women such as Margaret Stokes or "Mrs. T. Mitchell" provide significant examples of independent female travel. The fifth chapter is arguably one of the most stimulating parts of the study in terms of its firm focus and the material that it analyses. It investigates the rise of Irish pilgrimages to Catholic centres such as Lourdes, Oberammergau and Rome, highlighting the pilgrims' increased affiliation to a "universal" Roman Catholic Church, and examining Irish Protestant responses to these Catholic spaces. The role played by Thomas Cook's tourist agency is also significant. Accordingly, Chapter 3 is a welcome attempt to focus on the material conditions for travel and for the dissemination of travel writing, charting some of the sociological, infrastructural and technological changes that contributed to the development of Irish tourism abroad - most notably increased transport facilities and the rise of steam power.

As the author admits, such a broad undertaking necessarily means that the book cannot provide an exhaustive treatment of the topic. Many destinations, travellers, texts and themes have not been included. The focus is limited to a number of European sites, but one important debate about travel occurred regardless of specific destinations: a major motif in the discourse on Irish travel was the tension between home and abroad, and the encouragement to "see Ireland first". It is in this light, and against the background of a developing Irish tourist industry (notably in the rural, Gaelic-speaking west of Ireland) that Chapter 8 revisits Joyce's "The Dead" and the confrontation between Gabriel Conroy and Miss Ivors. Travel and discourse about travel bring about questions of identity - and in this case, a complex web of identities - which is really the main thread that ties the book together. No doubt it makes an important contribution to the fields of Irish studies and studies of European travel.

Michelle MiLan

Vincent Morley, The Popular Mind in Eighteenth-century Ireland, Cork University Press, 2017, ix +362 p, ISBN 978-1-7820-5208-1.

Vincent Morley est un historien irlandais, spécialiste du XvIII ${ }^{\mathrm{e}}$ siècle -the «long $18^{\text {th }}$ century » de 1691 à 1830 , depuis la conquête de l'Irlande par Guillaume III d'Orange jusqu'aux débuts de la campagne pour l'abrogation de l'Acte d'Union menée par Daniel O’Connell. Néanmoins l'ouvrage de Morley ne se donne pas 
comme objet d'étude les grands personnages de l'histoire irlandaise. Il entend en revanche mieux cerner la culture populaire irlandaise de l'époque par le biais d'un corpus de huit textes originaux. L'auteur en traduit sept pour la toute première fois en anglais. Que faire de l'encombrante langue de l'ancienne puissance coloniale? Comment rendre compte fidèlement du point de vue du " colonisé ", véhiculé par une oralité labile et fuyante en gaélique? La gageure et la prémisse pour cet ouvrage sont résumables ainsi : dans un souci de fidélité historique, on peut et on doit donner un accès aux non-irlandophones à ce que Morley appelle "l'esprit populaire irlandais ", ou autrement dit l'esprit des masses. Le paradoxe est alors triple : c'est par l'écrit et par l'anglais et enfin au $\mathrm{XxI}^{\mathrm{e}}$ siècle que l'auteur souhaite réévaluer et recentrer une culture gaélique orale $\mathrm{du} \mathrm{XvIII}^{\mathrm{e}}$ siècle. Les 8 textes présentés s'organisent autour de deux pôles: la poésie entre élégie et poèmes historiques pour la première partie et les chansons pour la seconde - pour certaines anonymes (chapitres 5 et 6). La qualité littéraire de l'ensemble du corpus peut laisser perplexe, comme l'avoue l'auteur lui-même en qualifiant un aisling censé éclairer le texte final sur la période O'Connellienne de " doggerel in both languages » (p. 305). L'aisling est cette forme poétique ou chantée, qui signifie en gaélique "vision » ou " rêve »selon la définition rappelée dans un glossaire bienvenu constitué par l'auteur, p. VIII. On notera enfin que l'auteur a organisé l'ouvrage autour de 8 thèmes présents dans les 8 textes rassemblés, thèmes pour la plupart déjà abordés dans ses recherches ou articles préalables : le royaume, la foi, la mémoire, la guerre, les patriotes, la terre, la rébellion et enfin en 1830, "Union? ", suivie d'un point d'interrogation évocateur. Au-delà du littéraire donc, le livre vaut en soi par l'ampleur de sa traduction du gaélique et la période couverte.

On remarquera aussi que l'entreprise de Morley fait écho de façon assumée à celle de Daniel Corkery dont l'ouvrage The Hidden Ireland (1924) citée dans l'introduction (pp.1-8) fournit ainsi le point de départ de l'analyse. Corkery souhaitait restituer une place à la poésie populaire irlandaise en gaélique. Son ouvrage était censé permettre la reconstruction de la vision du monde de la majorité irlandaise catholique. Celle-ci était dépeinte comme essentiellement pour ne pas dire exclusivement opprimée, rurale et dépossédée au XvıII ${ }^{\mathrm{e}}$ siècle. Selon Patrick Walsh, l'œuvre de Corkery fournit en son temps une forme de doxa au régime nationaliste irlandais après 1922. Morley, dans son ouvrage plus nuancé, vient souligner l'importance cruciale de la continuité et de la tradition portées par les masses au cours de l'histoire. On discerne là en filigrane l'influence de l'école historique du temps long portée en France naguère par Fernand Braudel. En Irlande pour Morley, ce temps long se fait linguistique et gaélique. Les masses irlandaises étaient majoritairement irlandophones avant 1800 dans l'île, fait généralement admis par les chercheurs mais insuffisamment pris en compte dans l'historiographie de l'Irlande. 
Si l'on ne peut que louer l'effort de réhabilitation objective de l'oralité illustré par l'ouvrage dans la propagation des idées dans une Irlande majoritairement analphabète, catholique et gaélique avant 1800 , on peut en revanche être plus sceptique à d'autres égards. Notamment quand l'auteur relègue le discontinu événementiel à une forme d'élitisme précisément et paradoxalement traditionnelle: " Far from being a mark of maturity, the historian's interest in highlighting apparent discontinuities is characteristic of a very traditional top-down approach to the Irish past " (p. 309). Ce qui est posé comme une évidence conservatrice par l'auteur: "Continuity is invariably more important than change for the great majority of people in any society " mériterait tout autant d'être questionné en nuance que la primauté supposée du discontinu.

C'est pourquoi au-delà des mérites indéniables d'un livre qui met en avant des sources méconnues, à une époque trop peu documentée dans la langue gaélique et pour cause -, on pourra comme Declan Kiberd émettre quelques réserves quant à la critique frontale opérée par Morley contre la pensée de Jürgen Habermas sur la culture de l'imprimé dans l'espace public (p. 311). Comme le rappelle Declan Kiberd ${ }^{1}$ à juste titre, la tradition orale fait souvent écho à la culture de l'imprimé : " any reader might be struck by the ways in which oral tradition can mimic print culture ". C'est ainsi souvent dans l'entre-deux et le mouvement dialectique plus que dans l'essence supposément stable, fût-elle méprisée, que l'on trouve des clefs en histoire. Mais ce serait ne pas rendre justice à l'originalité du propos de Morley que de le réduire à une pensée monologique. Ainsi le traitement de la religion catholique dans le chapitre 3 offre des analyses aussi riches et nouvelles que nuancées. Enfin, le livre est d'une clarté exemplaire sur la période considérée.

Thierry RoBIN

1. Declan Kiberd, "The Popular Mind in Eighteenth-century Ireland review: An elegant and luminous study", The Irish Times, [http://www.irishtimes.com/culturelbooks/the-popular-mind-in-eighteenth-century-ireland-review-anelegant-and-luminous-study-1.3032259], 15 avril 2017. 